\title{
Investigation of Third Gyro-harmonic Heating at HAARP Using Stimulated Radio Emissions and the MUIR and Kodiak Radars
}

\author{
A. Mahmoudian ${ }^{1, *}$ \\ The Bradley Department of Electrical and Computer Engineering, Virginia Tech, USA \\ W. A. Scales* \\ The Bradley Department of Electrical and Computer Engineering, Virginia Tech, USA \\ B. J. Watkins* \\ University of Alaska, Fairbanks, AK \\ P. A. Bernhardt* \\ Plasma Physics Division, Naval Research Laboratory, Washington D.C., USA \\ B. Isham, O.Vega-Cancel ${ }^{*}$
}

Department of Electrical and Computer Engineering, Inter American University of Puerto Rico, Bayamón, Puerto Rico

J. M. Ruohoniemi*

The Bradley Department of Electrical and Computer Engineering, Virginia Tech, USA

\begin{abstract}
This paper presents data from two campaigns at the High Frequency Active Auroral Research Program facility (HAARP) in 2011 and 2012. The measurements of stimulated radio emissions (often called stimulated electromagnetic emissions or SEE) were conducted $15 \mathrm{~km}$ from the HAARP site. The potential of Narrowband SEE (NSEE) as a new diagnostic tool to monitor artificial irregularities excited during HF-pump heating of the ionosphere is the main goal of this paper. This has been investigated using well established diagnostics including the Modular UHF Ionospheric Radar (MUIR) and Kodiak SuperDARN radars as well as Wideband SEE (WSEE). The measured data using these three diagnostics were compared to characterize the ionospheric parameters and study the plasma irregularities generated in the interaction region. Variation of the wideband/narrowband SEE features, SuperDARN echoes, and HF-enhanced ion lines (EHIL) were studied with pump power variation, pump frequency stepping near the third electron gyro-frequency $\left(3 f_{c e}\right)$ as well as changing beam angle relative to the magnetic zenith. In particular, electrostatic plasma waves and associated irregularities excited near the reflection resonance layer as well as the upper-hybrid resonance layer are investigated. The time evolution and growth rate of these irregularities are studied using the experimental observations. Close alignment of narrowband SEE (NSEE) with wideband SEE (WSEE) and EHIL was observed. SuperDARN radar echoes and WSEE also showed alignment as in previous investigations. Correlations between these three measurements underscore potential diagnostics by utilizing the NSEE spectrum to estimate ionospheric parameters such as electron temperature.
\end{abstract}

\section{Introduction}

Ionospheric modification experiments using high-power radiowaves have tremendous potential for diagnosing the Earths upper atmosphere, ionosphere, and magnetosphere. The transmitted electromagnetic (EM) wave interacts strongly

\footnotetext{
*Corresponding author

Email address: alirezam@vt.edu (A. Mahmoudian )

${ }^{1}$ phone number:+1-540-328-1127
}

with the ionosphere in the entire region from the plasma resonance down to well below the upper-hybrid (UH) resonance height. The reflection resonance layer is where the EM pump frequency $\left(f_{0}\right)$ equals the local plasma frequency $\left(f_{p e}\right)$. The $\mathrm{UH}$ resonance layer refers to the interaction altitude where $f_{0}$ is equal to the local UH frequency $\left(f_{U H}\right)$. The Langmuir-UH dispersion surface exhibits a resonance at all angles, between the plasma resonance parallel to the geomagnetic field to the $\mathrm{UH}$ resonance perpendicular to the geomagnetic field (e.g. Mjolhus et al., 2003). 
Ionospheric heating experiments have been recognized to be an ideal approach for investigation of plasma irregularity generation and evolution that can be observed by radar. An EM pump may produce electrostatic waves near the reflection altitude by a combined aperiodic and periodic three-wave parametric process (e.g. Gurevich, 2007). Due to the orientation of the pump electric field along the geomagnetic field near the reflection altitude, a standing Langmuir wave (LW) and spatially periodic but temporally aperiodic plasma density fluctuations are produced through an oscillating two-stream instability OTSI (or aperiodic process). The pump may also decays into lower frequency LWs and ion acoustic IA waves due to a three-wave parametric decay instability PDI (or periodic process). Multiple decays are referred to as wave cascading within the context of weak Langmuir turbulence. The downshift of the lower frequency LW is the IA wave frequency prescribed by the PDI frequency matching conditions. The pump-enhanced Langmuir waves (LW) processes give rise to HF-enhanced plasma lines (EHPL) and scatter from the IA fluctuations produced by PDI give rise to the HF-enhanced ion lines (EHIL) in the MUIR radar spectrum. The plasma density fluctuations with zero phase velocity generated through the OTSI process will appear in the MUIR spectrum with a zero frequency offset relative to the radar frequency. Both the PDI and OTSI occur close to the reflection height at approximately the same altitude. It has been shown that the OTSI has a higher threshold than PDI, but OTSI has a higher linear growth rate than the PDI (Stubbe, 1996; Fejer, 1988). Conversely, at the upper hybrid resonance altitude, the pump electric field is perpendicular to the geomagnetic field. The pump wave may resonantly excite perpendicularly propagating upper hybrid (UH) waves which become trapped in electron density reductions, forming a standing wave pattern, and ultimately result in growth of field aligned irregularities FAI through resonance instability (Vas'kov and Gurevich, 1977; Mjolhus et al., 2003). The field aligned structure is due to the more efficient transport along the geomagnetic field lines due to the relatively low electron collisionality in the F-region (e.g. Gurevich, 2007). These FAIs may be observed by SuperDARN radar (e.g. Hughes et al. (2003).

Stimulated radio emissions (also known as stimulated electromagnetic emissions or SEE) are secondary radio waves excited by a high-power HF (high-frequency) radio pump wave transmitted into the ionosphere. A new era of ionospheric remote sensing techniques resulted from the update of the HF transmitter at the High Frequency Active Auroral Research Program (HAARP) in 2007. Increasing the maximum transmitter power to 3.6 MW (effective radiated power $\mathrm{ERP} \sim 1 \mathrm{GW}$ ) has allowed studies of parametric decay instabilities responsible for SEE not previously possible. The classical SEE spectral lines with frequency offset by $1 \mathrm{kHz}$ up to $100 \mathrm{kHz}$ were identified and studied in detail in the 1980s and 1990s. There has been extensive study of physical processes and irregularities as- sociated with SEE spectral lines in a wide frequency band around the pump frequency $(1-100 \mathrm{kHz})$ during the last three decades (e.g. Leyser, 2001). For example, the downshifted maximum (DM), a distinct peak at a frequency approximately $10 \mathrm{kHz}$ downshifted from the pump frequency (Thide et al., 1982) results from nonlinear interaction of upper hybrid (UH) and lower hybrid (LH) waves (Leyser et al., 1989, 1990). The more rarely observed downshifted peak (DP) is a narrow emission downshifted by $1-3 \mathrm{kHz}$ from the pump frequency. The DP is very sensitive to small changes in $f_{0}$ and is only excited by $f_{0}$ near harmonics of electron gyro-frequency $n f_{c e}$ while the DM is not excited for $f_{0} \approx n f_{c e}$. The DP has been proposed to result from the parametric decay instability involving $\mathrm{UH}$ waves and IA waves at the first and strongest standing pump wave maximum below the reflection height (Huang and Kuo, 1995).

More recently narrowband sideband emissions (within $1 \mathrm{kHz}$ of pump frequency) of unprecedented strength have been reported during recent campaigns at HAARP, reaching up to $10 \mathrm{~dB}$ below the pump wave wave, and therefore by far are the strongest spectral features of secondary radiation that have been reported (Norin et al., 2009; Bernhardt et al., 2010; Fu et al., 2013; Mahmoudian et al., 2013a, 2014a, b; Scales, 2016). The emissions were shifted by only a few tens of Hertz from radio waves transmitted at several megahertz. Measurement of the narrowband SEE line spectrum excited in the ionosphere is expected to result in the development of new remote sensing techniques. The newly-discovered narrowband SEE (NSEE) features have not yet been studied to the same degree of detail as the classical SEE features. One of these new NSEE features results from Stimulated Brillouin Scatter (SBS). SBS is due to decay of the EM pump into another downshifted EM wave whose frequency down-shift is determined by a low frequency ion acoustic decay mode. It should be noted that SBS has been observed for the first time very recently at the European Incoherent Scatter Facility EISCAT (Fu et al., 2015). Previous studies by Bernhardt et al. (2009) and Mahmoudian et al. (2014a) have investigated the assesment of electron temperature using IA SBS lines.

So-called Magnetized Stimulated Brillouin Scatter (MSBS), which produces narrowband SEE, excited during ionospheric HF-pump heating at HAARP was observed for the first time by Bernhardt et al., (2009, 2010). MSBS process has an additional downshifted sideband due to a low-frequency Electrostatic Ion Cyclotron (EIC) decay mode as well as an IA low frequency decay mode. The EIC wave frequency is near the ion gyro-frequency $(\approx 50 \mathrm{~Hz})$ and produces a corresponding frequency shift in the MSBS line. The variation of MSBS lines with HF pump power, HF transmitter beam angle with respect to the direction of the background magnetic field, and with proximity of the HF pump frequency $f_{0}$ to the harmonics of electron gyrofrequency $n f_{c e}$, has been studied extensively over the past four years (Mahmoudian et al., 2013a; 2014a,b; Fu et al., 2013). 
The objective of this paper is to investigate the signatures of irregularities excited at the $\mathrm{UH}$ and reflection altitudes during HF pumping. Several diagnostics, including the UHF MUIR radar, the Kodiak Super-DARN backscatter radar, stimulated electromagnetic emissions detected on the ground, as well as a Digisonde, will be used to study the heated region of ionosphere at the UH and reflection resonance layers. Prompted by past observations, the focus of this work is to study narrowband SEE spectral lines (MSBS), wideband classical SEE (DP and DM), EHIL and OTSI lines in the MUIR spectrum as well as FAI measured by SuperDARN, and their interrelationship with heater beam angle, transmitter frequency, and HF pump power.

\section{Experimental Description}

The data presented in this paper was obtained during the July 2011 and August 2012 campaigns at HAARP $\left(62.39^{\circ} \mathrm{N}, 145.15^{\circ} \mathrm{W}\right)$ with maximum effective radiated power (ERP) of $1.0 \mathrm{GW}$ for the pump frequency $4.5 \mathrm{MHz}$. The SuperDARN radar located at Kodiak, Alaska was used to probe the ionospheric plasma at F-region heights above the heater. This HF radar detects backscatter when decameterscale irregularities are present. The MUIR radar located at the HAARP facility was used to study the time evolution of the HF-enhanced ion lines (EHIL) as well as OTSI line. SEE receiver and measurement setup are described in Mahmoudian et al., (2013b).

The ultrahigh frequency (UHF) diagnostic radar called MUIR was added to the HAARP facility in 2005 and is located about $2 \mathrm{~km}$ from the HF antenna. The spectra of plasma waves generated by the HF pump at the reflection altitude may be computed from the backscattered echoes measured by MUIR. The presence of irregularities may be deduced from the spectra shapes and altitude profile of radar echoes (Oyama et al., 2005). The plasma waves associated with the OTSI and EHIL lines discussed earlier may be observed with MUIR radar. The OTSI is at zero frequency offset relative to the radar frequency and is indicative of the plasma density perturbation (which have zero phase velocity) while EHILs are offset by $5 \mathrm{kHz}$ above and below the OTSI line frequency due to the upward and downward propagating IA waves. MUIR radar has only one receiver channel and can be tuned to EHIL and OTSI features with frequency span about $+/-10 \mathrm{kHz}$, or to several $\mathrm{MHz}$ to see Langmuir waves. The first observation of the enhanced plasma lines during ionospheric heating experiments at HAARP using the MUIR radar was reported by Oyama et al., (2005, 2006). Burton et al., (2008) used the MUIR radar to study cascading Langmuir waves excited by the parametric decay instability. Cohen et al. (2010) used MUIR to investigate artificial ionospheric ducts which are sheet-like large-scale plasma irregularities and excited by O-mode and X-mode HF heater waves via thermal filamentation instabilities within and orthogonal to meridional planes, respectively.

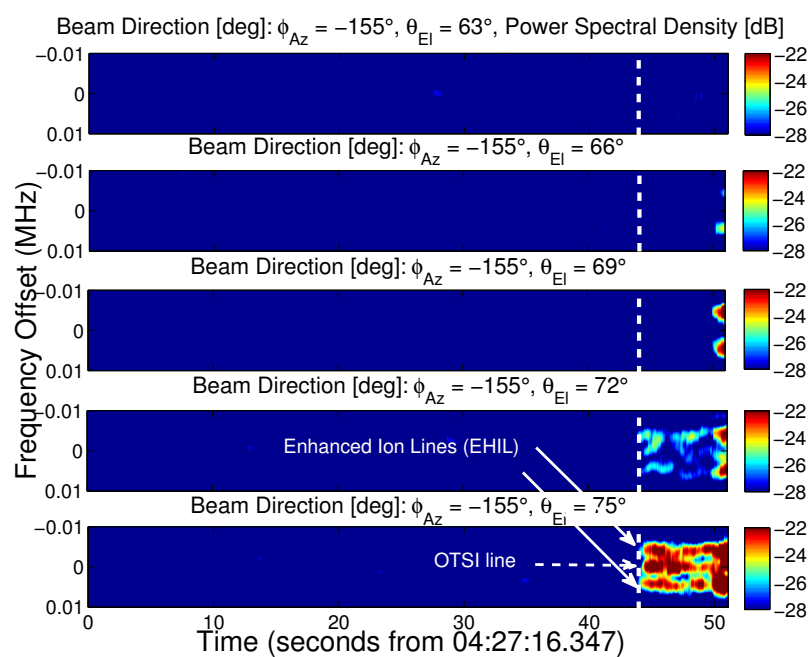

Beam Direction [deg]: $\phi_{A z}=-155^{\circ}, \theta_{E \mid}=63^{\circ}$, Power Spectral Density [dB]
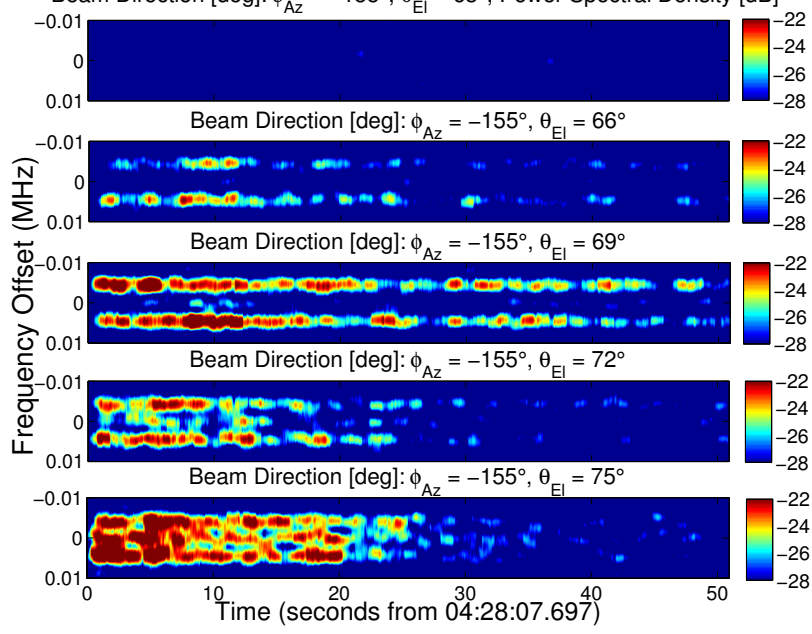

Beam Direction [deg]: $\phi_{A z}=-155^{\circ}, \theta_{C \mid}=63^{\circ}$, Power Spectral Density [dB]

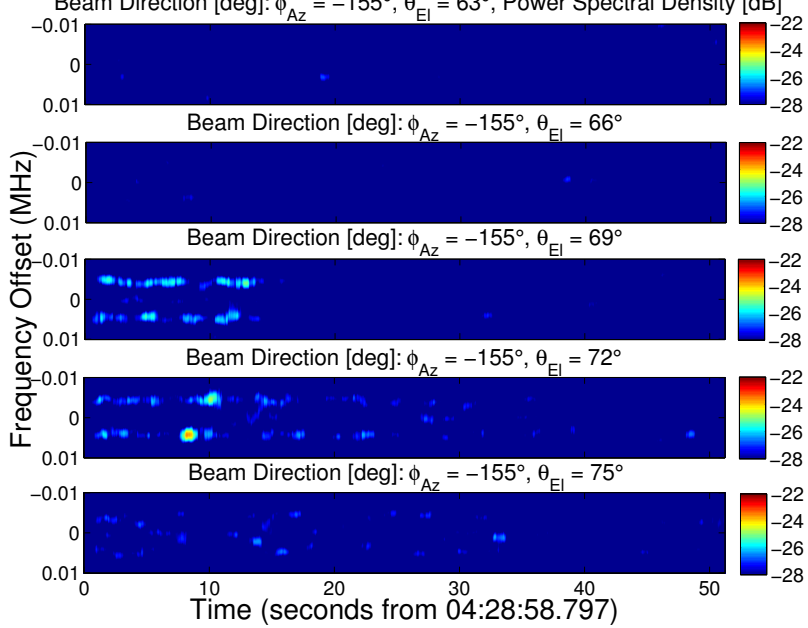

Figure 1: Time evolution of HF-enhanced ion lines and of the OTSI line measured by the MUIR radar at $63^{\circ}, 66^{\circ}, 69^{\circ}, 72^{\circ}$ and $75^{\circ}$ elevation angles. This corresponds to zenith angles of $27^{\circ}, 24^{\circ}, 21^{\circ}$, $18^{\circ}$, and $15^{\circ}$, respectively. The heater beam was pointed to a zenith angles of $14^{\circ}$. The horizontal axis shows the time, and the vertical axis shows the offset frequency, for each line. The panels are consecutive during the HF-on period. The heater was turned on at 4:28 UT and turned off at 4:32 UT. The HF pump frequency was set to $4.3 \mathrm{MHz}$. Vertical dashed line shows the start of the heating cycle. 

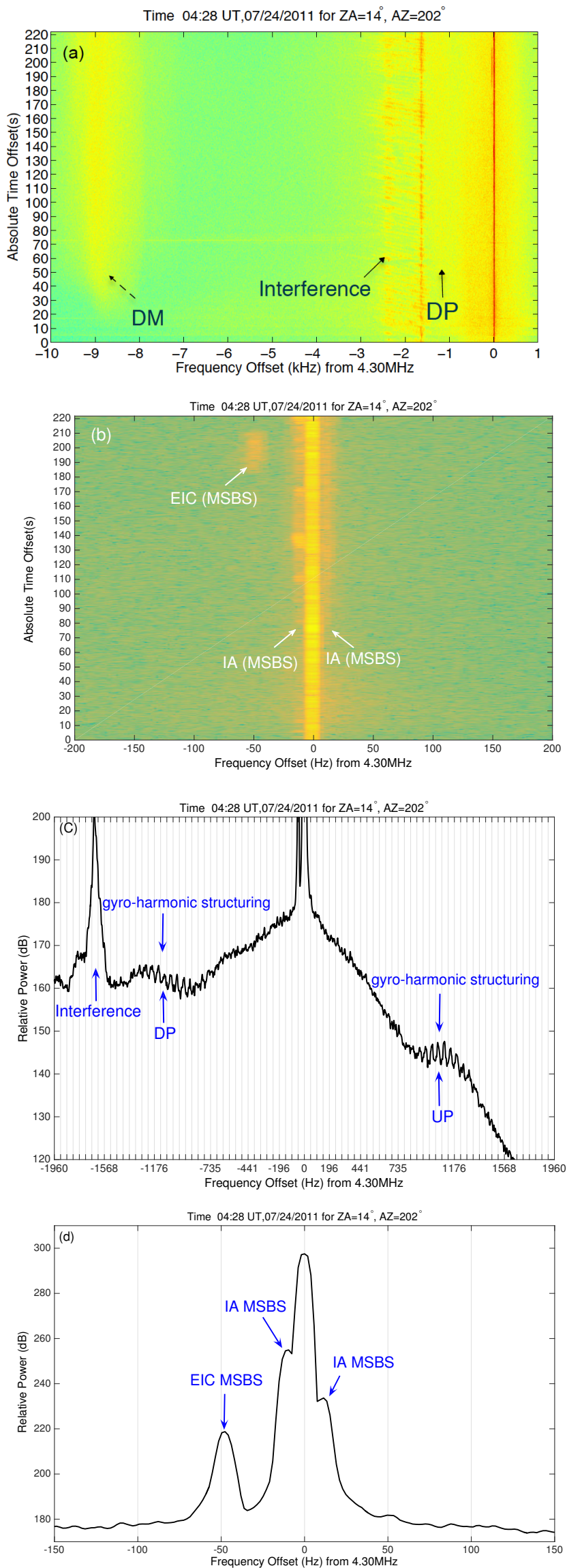

Figure 2: Time history of the spectra of a) wideband SEE (WSEE) and b) narrowband SEE associated with Figure 1. The pump power 4 increased from $0.1 \mathrm{MW}$ at $\mathrm{t}=0 \mathrm{~s}$ in $0.1 \mathrm{MW}$ steps every $6 \mathrm{~s}$. c) Spectra averaged stimulated ion Bernstein scatter (SIBS) embedded in the downshifted peak (DP) d) Spectra averaged of IA MSBS and EIC MSBS lines.
Beam Direction [deg]: $\phi_{A z}=-155^{\circ}, \theta=63^{\circ}$, Power Spectral Density [dB]

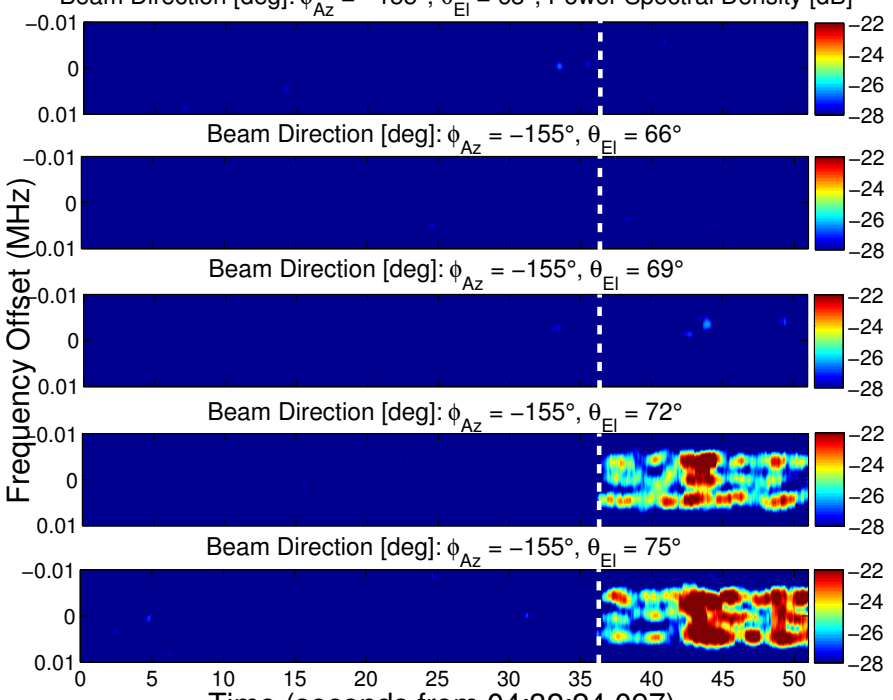

Time (seconds from 04:32:24.097)

Beam Direction [deg]: $\phi_{\mathrm{Az}}=-155^{\circ}, \theta_{\mathrm{El}}=63^{\circ}$, Power Spectral Density [dB]

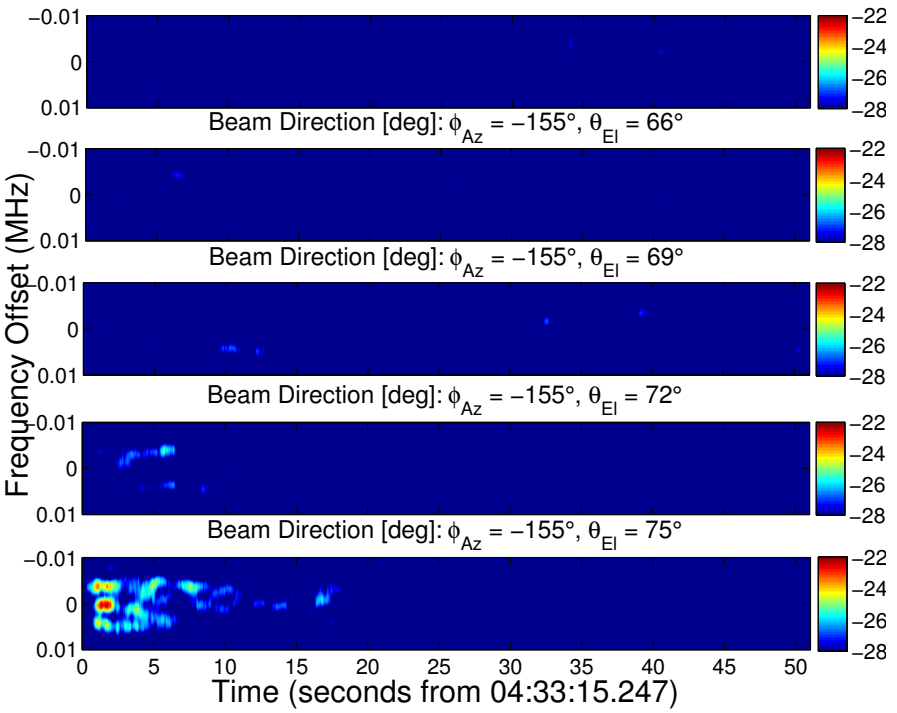

Figure 3: Time history of HF-enhanced ion and OTSI lines observed by UHF radar at HAARP for the HF beam pointed at zenith angle $18^{\circ}$. Other parameters the same as Figure 1. The panels are consecutive during the HF-on period. The heater was turned on at 4:33 UT (shown with vertical dashed lines) and turned off at 4:37 UT. Vertical dashed line shows start of heating cycle. 
An important complementary diagnostic for observing waves and irregularities during heating is the SuperDARN HF radar. The Kodiac SuperDARN radar has been used to interpret the signatures of electrostatic ES fluctuations generated within the heated volume above HAARP at the UH altitude (e.g. Hughes et al., 2003, 2004). Due to the mutual geometry of HAARP and of the Kodiak SuperDARN radar, the signal observed by the Kodiak radar can result only from electron density fluctuations in the direction normal to the geomagnetic field.

The campaign on July 24, 2011 from 4:28 UT to 4:58 UT considered pump power stepping for pump frequency $f_{0}$ near the third electron gyro-harmonic to measure the pump power required to excite the narrowband SEE (NSEE) emission lines as well as of artificial field aligned irregularities (FAI). The power was increased from 0.1 MW (corresponding to an effective radiated power (ERP) $27 \mathrm{MW}$ ) to $3.6 \mathrm{MW}$ (ERP $1 \mathrm{GW})$ in $0.1 \mathrm{MW}$ steps every $6 \mathrm{~s}$. The total heating cycle for this experiment was 5 min including $4 \mathrm{~min}$ heating and $1 \mathrm{~min}$ off period. The HAARP transmitter was operated in O-mode polarization during all experiments. The beam was transmitted in a quasicontinuous wave mode. The direction of the HAARP HF beam can be swept in the meridian plane of the magnetic field from the magnetic zenith case $\left(14^{\circ}\right.$ zenith) to $\pm 30^{\circ}$ zenith. It should be noted that the HAARP beamwidth is of the order of $14^{\circ} \times 10^{\circ}$ for pump frequencies near $3 f_{c e}$ (and beamwidth is about $5.7^{\circ} \times 4.5^{\circ}$ for $f_{\text {pump }}=10 \mathrm{MHz}$ ). The experiments conducted in 2011 campaign were set up for zenith angles $14^{\circ}, 18^{\circ}, 21^{\circ}, 24^{\circ}, 27^{\circ}$, and $30^{\circ}$. The heater beam angle was changed during the $1 \mathrm{~min}$ "off" period. The MUIR diagnostic radar beam was operated with 5 elevation angle positions: 63, 66, 69, 72, 75 degrees. Coded pulses of length 996 micro-seconds, with 4 microseconds baud lengths, were used, thus yielding a range resolution of 600 meters. The inter-pulse period (IPP) was 10 ms. For the experiments where five directions were used and single pulses were transmitted sequentially in multiple positions, the effective IPP in each direction was $50 \mathrm{~ms}$.

During all 2012 experiments, the HAARP heater was operated transmitting O-mode continuous wave. The experiments were carried out on 9 August 2012 between 3:30 UT and 4:00 UT, and between 10:30 UT and 11:00 UT. These experiments aimed to study the effect of the proximity of the pump frequency to the second and third electron gyro-frequencies. Only results for $3 f_{c e}$ heating will be discussed here. The pump frequency was also tuned to 4.26 $\mathrm{MHz}$ for $30 \mathrm{~s}$ at 3:35 UT. The pump frequency was then increased from 4.28 to $4.38 \mathrm{MHz}$ in $20 \mathrm{kHz}$ steps during 45-s "on" and 45-s "off" periods between 3:36 to 3:45 UT when the local peak plasma frequency was $4.5 \mathrm{MHz}$ as determined by the HAARP digisonde on site. During this experiment the HF transmitter at HAARP was pointed at magnetic zenith (MZ). This experiment was repeated between 3:45 UT to 3:55 UT for the transmitter beam pointed at zenith angle $(\mathrm{ZA})=21^{\circ}$. The azimuth angle was $200^{\circ}$ for all cases. The MUIR radar phased-array an- tenna was directed along the magnetic zenith.

\section{Experimental Results}

\subsection{Pump power variation}

The experiment on 24 July 2011 from 4:28 UT to 4:58 UT (20:28-20:58 Alaska daylight time) was aimed at examining the effect of the pump power. The transmitter was operated with 4-min on and 1-min off cycles. The Omode $\mathrm{HF}$ beam was pointed to the azimuth angle of $202^{\circ}$ and zenith angle was varied from $14^{\circ}$ (along the magnetic field lines or magnetic zenith $\mathrm{MZ}$ ) to $30^{\circ}$ in five steps.

Figure 1 shows the time evolution of EHIL (with frequency offset $5 \mathrm{kHz}$ ) and OTSI line measured by the MUIR radar at elevation angles $\theta_{E L}=63^{\circ}, 66^{\circ}, 69^{\circ}, 72^{\circ}$, and $75^{\circ}$. The pump power was stepped from $0.1 \mathrm{MW}$ to 3.6 MW every $6 \mathrm{~s}$ in $0.1 \mathrm{MW}$ steps. It should be noted that the angles shown in figures 1,3 , and 5 refer to the elevation angle of the MUIR radar. The magnetic zenith angle at that time corresponded to approximately azimuth 202 and elevation 76 degrees. The MUIR measurement closest to the magnetic zenith is $\theta_{E L}=75$. In figure 1 , the $\mathrm{HF}$ heater was directed to the magnetic zenith (or very close) and as expected there is a strong MUIR radar response when the radar looks to the magnetic zenith. The vertical axis shows the offset frequency with respect to radar frequency. The OTSI peak is observed at the radar frequency $446 \mathrm{MHz}$ which is set to zero in the figure. Clear enhancement in the spectrum magnitude can be seen (not shown) in the range from $220-280 \mathrm{~km}$. The HF transmitter was tuned to $4.3 \mathrm{MHz}$ and turned on at 4:28 UT for the beam zenith angle $14^{\circ}$. The heater was turned off at 4:32 UT. The MUIR data shows strongly-enhanced signal returns just after turn-on of the HF pump wave. This is known as the "overshoot". A weaker persistent enhancement was observed for the remainder of the heating cycle. As can be seen, HF-enhanced ion lines (EHIL) appear at larger elevation angles first (closer to magnetic zenith) and have higher intensity. As the heating continues they appear at elevation angles further away from the magnetic zenith. The OTSI line only appears for $\theta_{E L}=75^{\circ}$ and $72^{\circ}$. The time delay between the formation of EHIL at $\theta_{E L}=75^{\circ}$ and $69^{\circ}$ is about 5 seconds.

Figure 2 shows the time history of narrowband and wideband SEE spectra associated with Figure 1. According to the ionogram data, the altitude of HF reflection was around $230 \mathrm{~km}$ during this experiment. The International Geomagnetic Reference Field (IGRF) model provided the magnetic field strength and direction in the upper atmosphere over HAARP. The magnetic field near the HF reflection altitude is estimated to be $|B|=5.082 \times 10^{-5} \mathrm{~T}$ which results in $3 f_{c e}$ approximately equal to $4.27 \mathrm{MHz}$. The WSEE spectra in Fig 2a show that the DM appeared for pump power higher than $30 \mathrm{MW}$ ERP (30 s after heater turn-on). It should be noted that within the $6 \mathrm{~s}$ dwell time on each power level, the plasma processes in general may 


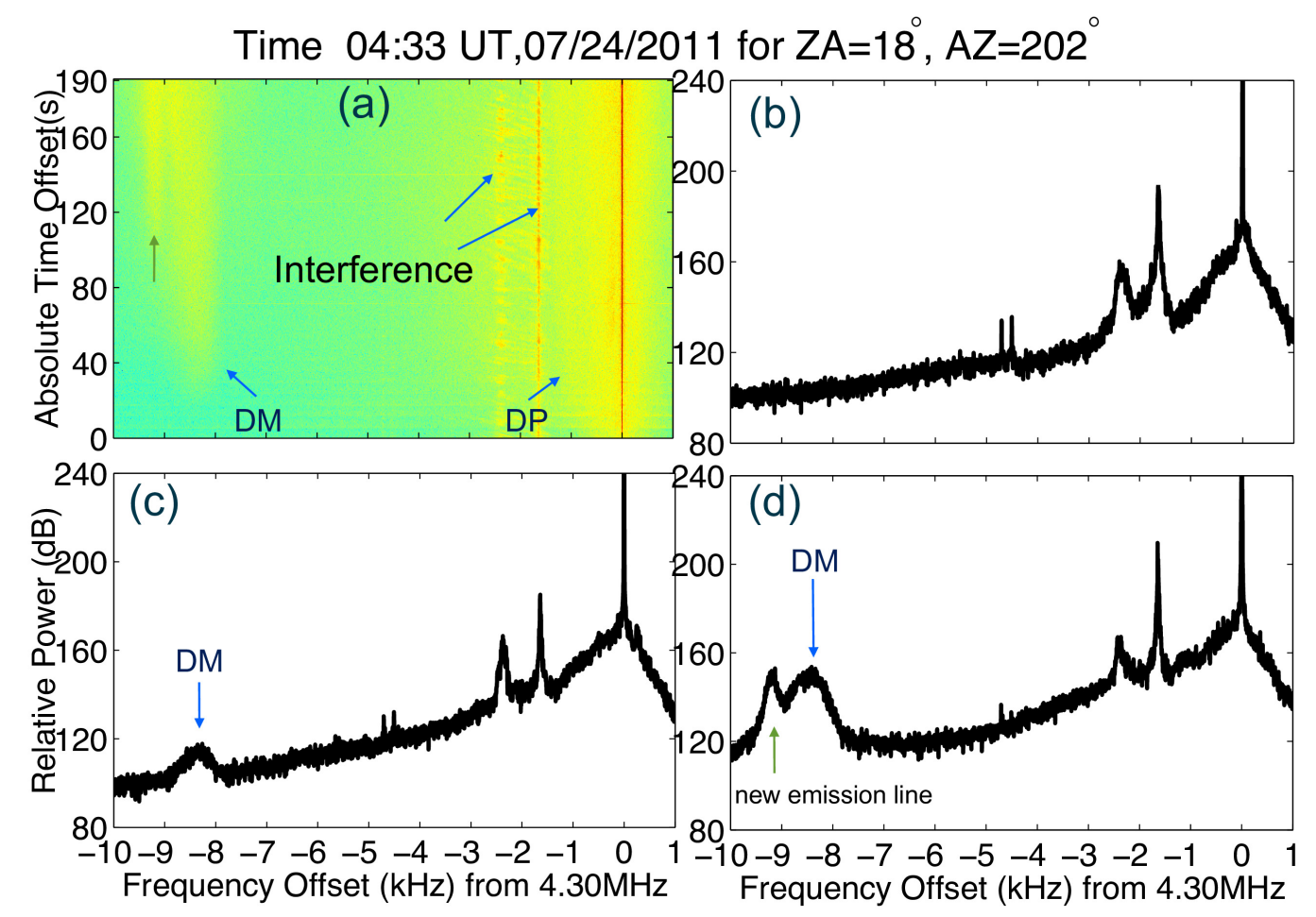

Figure 4: a) Time history of wideband SEE features for the HF beam pointed at zenith angle $18^{\circ}$, corresponding to Figure 3 . Snapshots are shown at b) t=10 s, c) t=80 s, d) t=150 s. Panels (a) and (c) show the new emission line observed at $9.1 \mathrm{kHz}$ (green arrow).

not have time to reach steady state, at least not at the lower power levels (Leyser et al., 1994).

NSEE lines resulting from MSBS at the reflection resonance altitude include Ion acoustic (IA) emission lines with a frequency offset of $\approx 10 \mathrm{~Hz}$, and electrostatic ion cyclotron (EIC) emission lines with frequency shifts of about $50 \mathrm{~Hz}$ from the pump frequency and are consistent with the MSBS matching condition (Bernhardt et al., 2009; Bernhardt et al., 2010; Mahmoudian et al., 2013a, 2014b). The EIC line with frequency offset $50 \mathrm{~Hz}$ and the IA line with offset $\sim 10 \mathrm{~Hz}$ (due to MSBS) appear for pump powers higher than $3 \mathrm{MW}$ and $0.7 \mathrm{MW}$, respectively. Figure 2c shows the first observations of embedded gyro-harmonic spectral structuring in the DP. These absorption bands are observed near harmonics of the $\mathrm{O}^{+}$ ( gyrofrequency $(\sim 50 \mathrm{~Hz})$. This spectral structuring is due to merging of ion acoustic and neutralized ion Bernstein wave modes. It should be noted that parametric decay instabilities near the upper-hybrid interaction altitude in which the pump field ultimately decays into high frequency upper hybrid/electron Bernstein and low frequency neutralized ion Bernstein IB and/or obliquely propagating ion acoustic waves is called Stimulated Ion Bernstein Scatter (SIBS) (Samimi et al., 2012; Mahmoudian et al., $2013 \mathrm{~b})$. The neutralized ion Bernstein waves propagate slightly off perpendicular $\left(k / k_{\perp}>\sqrt{m_{e} / m_{i}}\right)$ and have different dispersive characteristics due to Boltzmann electron behavior in comparision with the pure ion Bernstein mode which propagates virtually perpendicular to the magnetic field (e.g. Chen, 2012). Previous theoretical modeling by Mahmoudian et al. (2013b) predicted this behavior. This spectral characteristic was first observed and theoretically modeled for heating near $2 f_{c e}$ at HAARP by Samimi et al. (2012).

The formats of Figure 3 and 5, and the corresponding experimental setups, are the same as in Figure 1, except the HF pump pointed at zenith angles $18^{\circ}$ and $30^{\circ}$, respectively. The associated SEE spectrograms obtained during these two experiments are shown in Figures 4 and 6 . The formation of EHIL and OTSI in Figure 3 shows a similar trend to Figure 1. This is also expected since the HFpump beam angle only varies 4 degrees between the two cases. As can be seen in Figure 3, the OTSI line lasts for 15 seconds after heater turn-on, in comparison to Figure 1 , which shows the presence of the OTSI line for $35 \mathrm{~s}$ after heater turn-on. The HF-enhanced ion lines (EHIL) develop at lower elevation angles (i.e. further away from the magnetic field and vertical directions) as shown in Figure 5 , which is expected from the HF beam pointed at higher angles relative to magnetic zenith $\left(\mathrm{ZA}=30^{\circ}\right)$. EHIL and OTSI lines appear at $\theta_{E L}=72^{\circ}$ and $75^{\circ}$ at later times during the heating cycle. The time evolution of wideband SEE spectrum associated with Figure 3 is shown in Figure 4. MSBS-associated IA (not shown in Figure 4) and DM lines start to develop as the HF pump power increases to values larger than $0.5 \mathrm{MW}$ and $0.9 \mathrm{MW}$, respectively. The SEE spectra in Figure 4 also show a new emission line that develops for pump powers higher than 1.2 MW. The new 


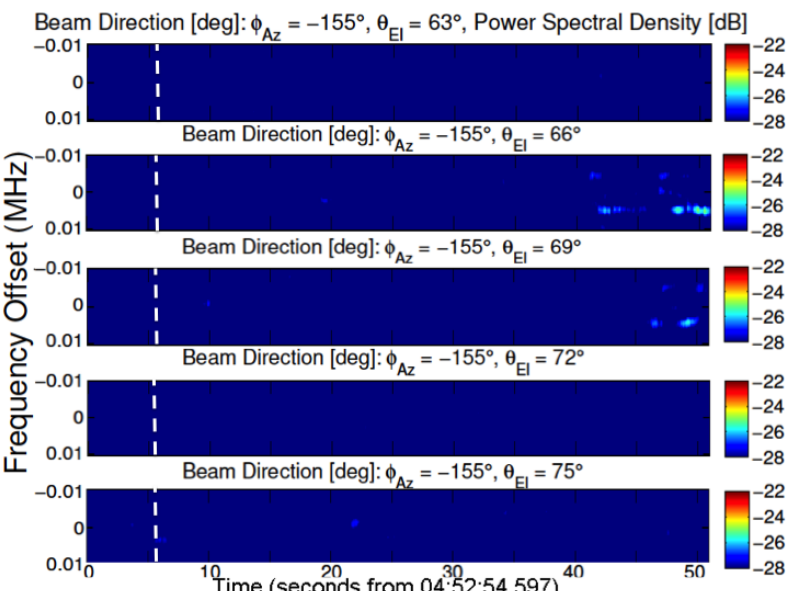

Time (seconds from 04:52:54.597) ${ }^{40}$ Beam Direction [deg]: $\phi_{A z}=-155^{\circ}, \theta_{E 1}=63^{\circ}$, Power Spectral Density [dB]
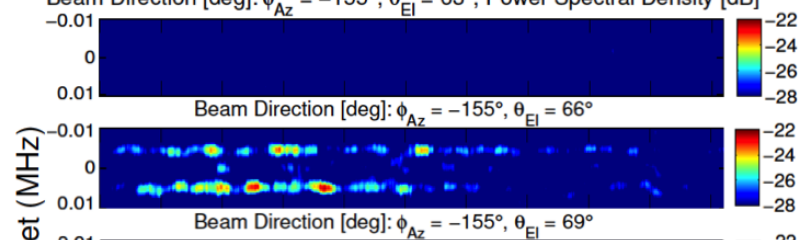

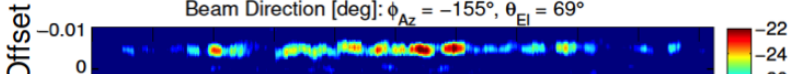

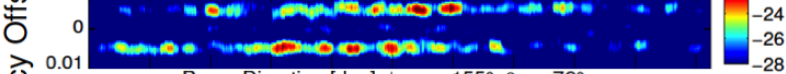

글 Beam Direction [deg]: $\phi_{A z}=-155^{\circ}, \theta_{E !}=72^{\circ}$
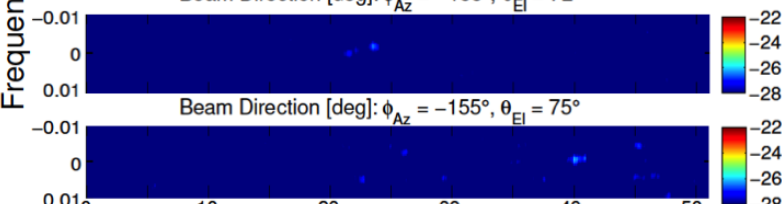

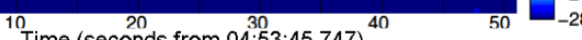

Beam Direction [deg]: $\phi_{A z}=-155^{\circ}, \theta_{\mathrm{EI}}=63^{\circ}$, Power Spectral Density [dB]
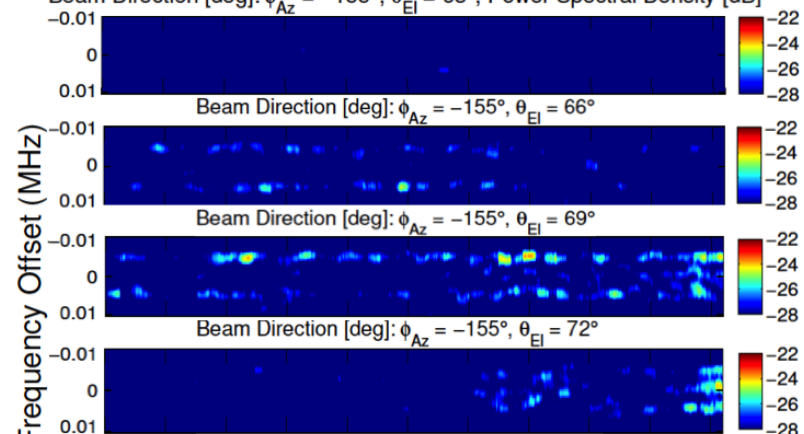

亡े 0.01 -

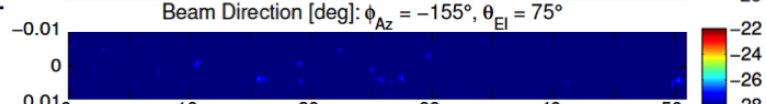

${ }^{10}{ }^{20}{ }^{30}{ }^{30}{ }^{40}{ }^{40}$

Beam Direction [deg]: $\phi_{A z}=-155^{\circ}, \theta_{\mathrm{EI}}=63^{\circ}$, Power Spectral Density [dB]
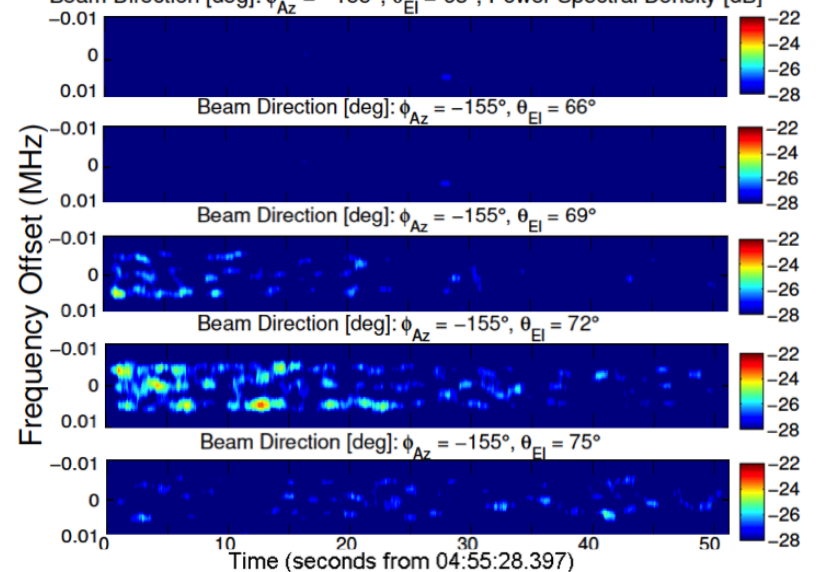

Figure 5: Similar to Figure 1, for the heater beam pointed to zenith angles $30^{\circ}$. The heater was turned on at 4:53 UT and turned off at 4:57 UT. The panels are consecutive during the HF-on period. Vertical dashed line shows the start of the heating cycle.
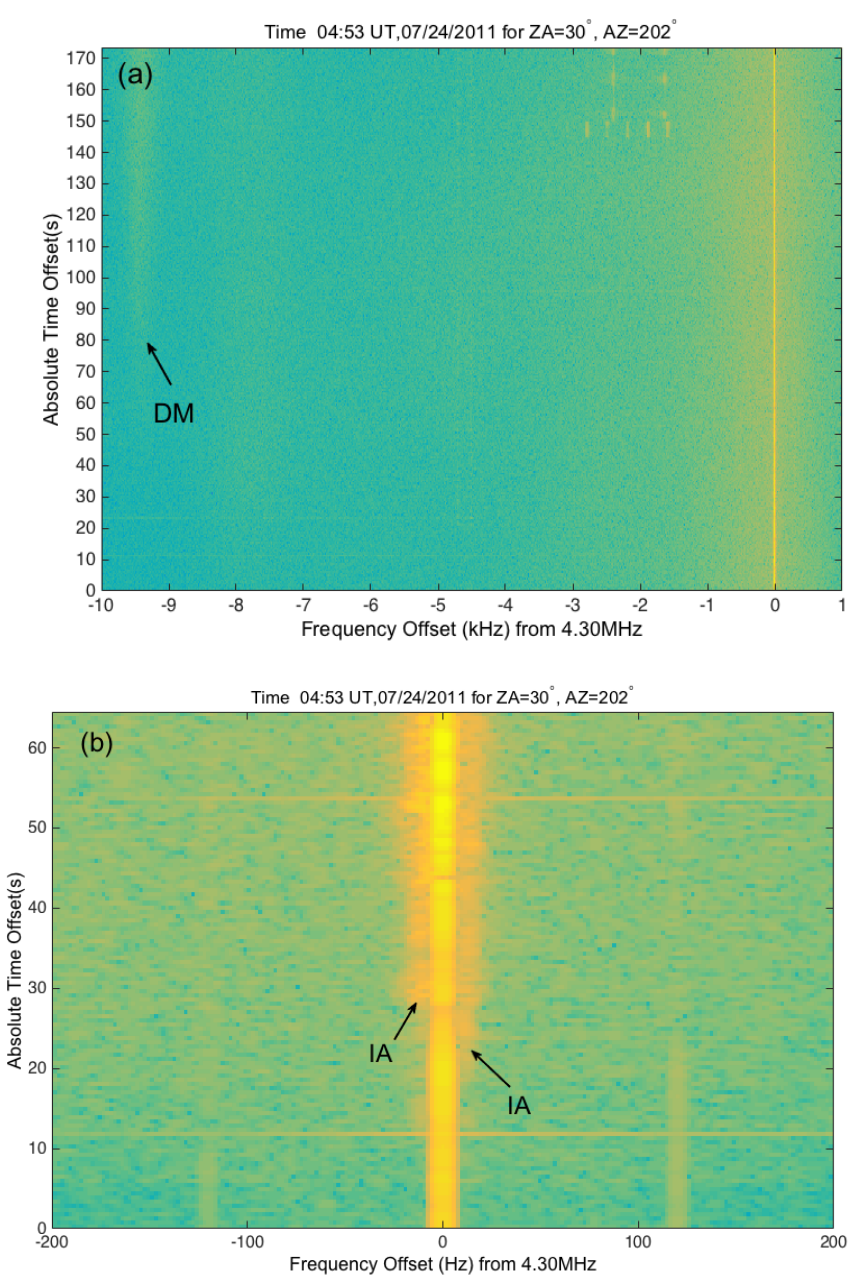

Figure 6: Time history of the SEE spectra of (a) wideband SEE features b) narrowband SEE features associated with Figure 5. The pump power increased from $0.1 \mathrm{MW}$ at $\mathrm{t}=0 \mathrm{~s}$ in $0.1 \mathrm{MW}$ steps every $6 \mathrm{~s}$. The maximum value of power at end of cycle at $170 \mathrm{~s}$ is $2.8 \mathrm{MW}$.

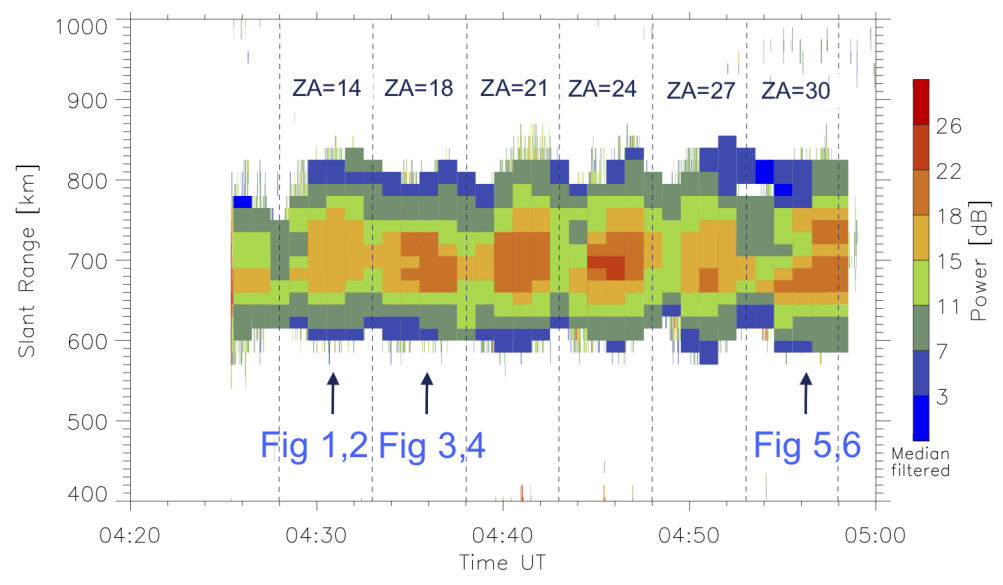

Figure 7: Backscatter from artificial irregularities observed by the Kodiak radar during ionospheric heating on 24 July 2011. The beam angle was pointed at zenith angles $14^{\circ}, 18^{\circ}, 21^{\circ}, 24^{\circ}, 27^{\circ}$ and $30^{\circ}$, and pump power was stepped from 0.1 MW to 3.6 MW. Arrows denote the heating cycles associated with Figures 1 to 6. 
emission line has a higher threshold relative to the well known DM and appears at $\Delta f \sim 9.1 \mathrm{kHz}$. The new SEE line shows a similar behavior to the split DM discussed by Leyser et al. (1990).

Figure 7 shows the echoes observed by the Kodiak radar, when the HAARP transmitter was operating at 4.3 $\mathrm{MHz}$, for six aspect angles $\left(14^{\circ}, 18^{\circ}, 21^{\circ}, 24^{\circ}, 27^{\circ}\right.$, and $30^{\circ}$ ), in the time period 4:28 to $4: 58$ UT on July 24,2011 , corresponding to Figures 1 to 6 . The transmitter beam remained at each angle for 5 min while the pump power was increased from 0.1-MW to 3.6 MW in 0.1 MW steps every 6 $\mathrm{s}$ (a total of $4 \mathrm{~min}$ ) and 1-min off period. The figure shows the formation of FAIs and stronger SuperDARN echoes as the pump power increases. The irregularities take about a minute to form in all HF beam angles, which corresponds to the pump power $1 \mathrm{MW}$.

\subsection{Frequency stepping near the $3^{\text {rd }}$ gyro-harmonic}

On 9 August 2012, the HAARP facility was operated near the third electron gyro-harmonic and the beam was pointed towards the magnetic zenith $\left(202^{\circ}\right.$ azimuth, $14^{\circ}$ zenith) and also (200 azimuth, $21^{\circ}$ zenith). Each heating cycle was $10 \mathrm{~min}$, with pump frequency variation from 4.26 $\mathrm{MHz}$ to $4.38 \mathrm{MHz}$ in 7 steps of $20 \mathrm{kHz}$ each.

Figure 8 shows the HF-enhanced ion and OTSI lines in the MUIR radar spectrum for pump frequencies 4.26 , $4.28,4.3,4.32,4.34$ and $4.36 \mathrm{MHz} .3 f_{c e}$ is approximately equal to $4.31 \mathrm{MHz}$ during this experiment. Persistent ion lines were observed for pump frequencies 4.3 and $4.32 \mathrm{MHz}$ which is near $3 f_{c e}$. Wideband and narrowband SEE observations corresponding to Figure 8 are presented in Figure 9. The variation of two wideband SEE features (DM and DP) with changes in the HF pump frequency near $3 f_{c e}$ is shown in Figure 9a. The EIC MSBS line shown in Figure $9 \mathrm{~b}$ is much weaker than the IA MSBS line and appears in a wider frequency band. The EIC line disappears first as the pump frequency approaches $3 f_{c e}$, which is consistent with the data presented in Mahmoudian et al. (2014a). Backscatter recorded by the Kodiak SuperDARN radar signal during the heating experiment on 9 August 2012 is shown in Figure 10. The observations show the supression of excited FAI for pump frequencies close to $3 f_{c e}$, which is consistent with previous observations (Mahmoudian et al., 2013b). As can be seen in Figure 10, the FAI irregularities observed by the Kodiak radar are strongest for the $\mathrm{HF}$ beam pointed at zenith angle $=21^{\circ}(3: 45-3: 55 \mathrm{UT})$. They also appear in a broader spatial range in comparison to pump heating along the magnetic zenith (3:35-3:45 UT).

\section{Discussion}

There are two processes that result in density variations at half the wavelength of the MUIR radar as noted earlier. One of these is the parametric decay instability PDI which results in Langmuir (LW) and ion-acoustic (IA) waves propagating along the geomagnetic field at the reflection height. The plasma density variations due to the IA waves are observed by MUIR in the form of EHIL (shown in Fig 1, 3, and 5). The other process is the oscillating two-stream instability (OTSI) which produces electron density perturbation together with standing Langmuir waves. MUIR detects these waves at zero Doppler due to the stationary standing wave pattern known as OTSI line. The EHIL is offset from the OTSI line due to the IA phase velocity. As shown in Figures 1, 3, and 5 , the OTSI line appears with zero frequency offset relative to the MUIR radar frequency and is stronger for angles close to magnetic zenith. It should be noted that both of these processes (OTSI and PDI) occur close to the reflection height at approximately the same altitude. The strongest excitations would be expected closer to the vertical, since the pump electric field component parallel to the geomagnetic field increases in the reflection region. Observations show the development of the OTSI line in the MUIR spectra in a short timescale of the order of few miliseconds. According to Figures 1 and 3, OTSI lines appear in the MUIR spectrum at the lowest pump power 0.1 MW for pump heating near magnetic zenith $\left(\mathrm{ZA}=14^{\circ}\right.$ and $\left.18^{\circ}\right)$. The pump power $0.1 \mathrm{MW}$ corresponds to an electric field amplitude of the order of $250 \mathrm{mV} / \mathrm{m}$ at the resonance layer in the F region (Mahmoudian et al., 2013a). This is based on the WKB approximation and D-region absorption has not been considered in this calculation. Theoretical calculations by Fejer (1988) has predicted a growth time for PDI and OTSI processes of the order of $10 \mathrm{~ms}$ for pump amplitude of $0.4 \mathrm{~V} / \mathrm{m}$. It should be noted that the amplitude of enhanced electric field near the reflection amplitude decreases for HF beam poined at larger angles relative to the magnetic field (Lundborg and ThidE, 1985). Therefore, an increase in the excitation time of the OTSI is expected as the HF beam was tilted to angles further away from magnetic zenith which is consistent with the theory of the OTSI (Fejer 1988). This is in agreement with the experimental observations shown in Figure 5 which shows the excitation of OTSI line at later times in the heating cycle in comparision with Figures 1 and 3.

The EHIL in the MUIR radar spectrum due to HF heating was presented in Figures 1, 3 and 5. Enhancement of the HF pump-induced ion line measured with the MUIR radar at HAARP shows alignment with the narrowband SEE IA spectral line for the pump power and beam angle variation as shown in Figures 1 to 6 . The MUIR spectrum ion-acoustic peaks are shifted above and below $f_{\text {radar }}$ by the ion acoustic frequency. It should be noted that according to Figure 9, the spectral amplitude of the DP and IA lines both disappear in the SEE spectra near the same pump frequency relative to $3 f_{c e}$. DP and IA MSBS lines both involve IA waves and as a result qualitatively similar behavior during pump frequency variation near $3 f_{c e}$ as was observed in EISCAT experiments (Fu et al., 2015). The correlation of the EHIL observed by the MUIR radar and narrowband SEE spectra (IA lines produced by the MSBS process) is observed for pump fre- 


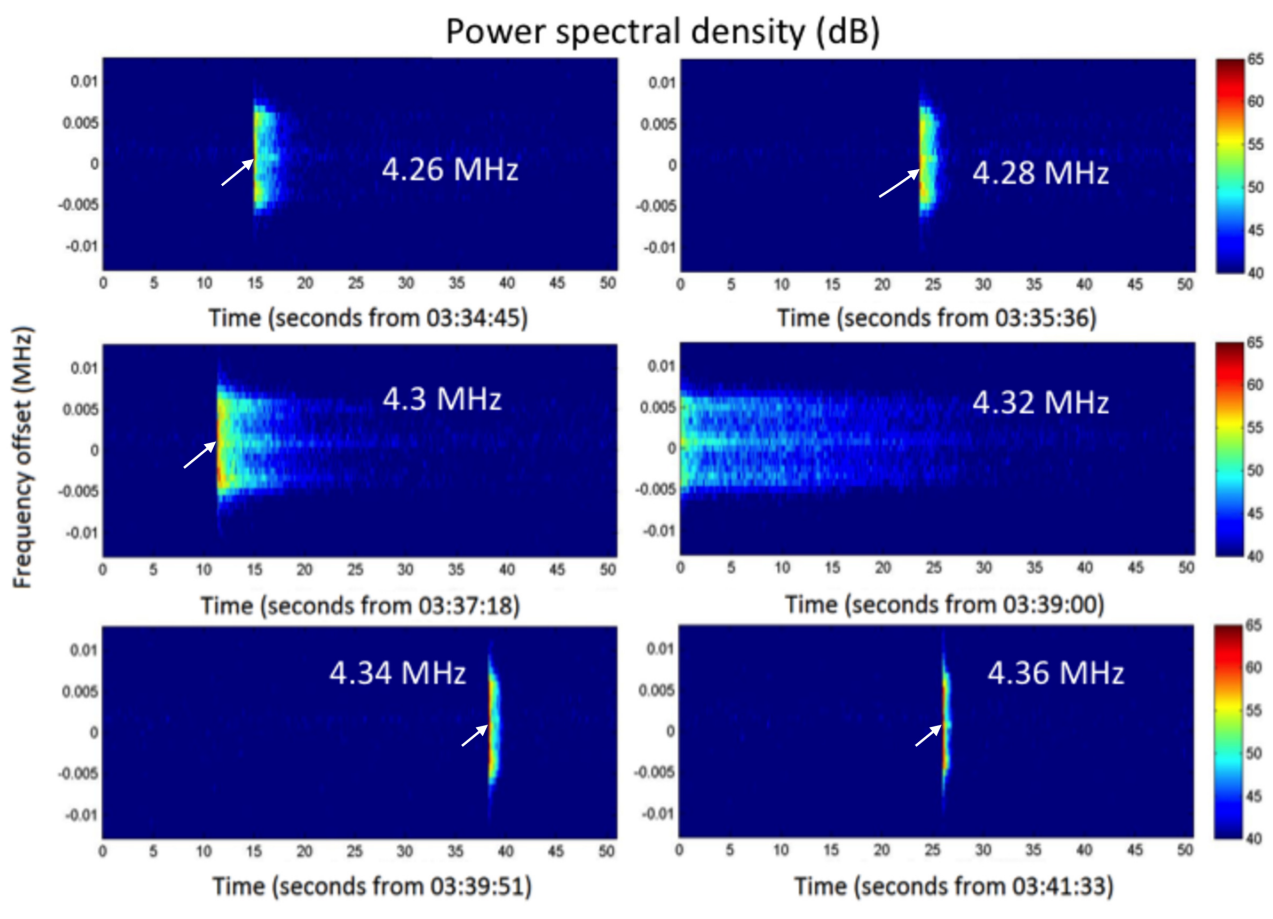

Figure 8: UHF radar observations of HF-enhanced ion-line (EHIL) structures associated with pump frequency stepping near $3 \mathrm{f}_{\text {ce }}$. The experiment was conducted on 9 August 2012. The white arrows show the beginning of the heating cycle. $3 f_{c e}$ was around 4.31 MHz during this experiment.

quency variations near $3 f_{c e}$. A close comparison of the variation in strength of EHIL and IA MSBS lines with pump frequency shows a correlation. According to Figure 9 , the most pronounced and strongest IA MSBS lines are observed for pump frequencies $4.28 \mathrm{MHz}$ and $4.3 \mathrm{MHz}$. As can be seen in Figure 8, the strongest EHIL was observed for pump frequencies $4.28 \mathrm{MHz}$ and $4.3 \mathrm{MHz}$ as well. A weaker IA MSBS line excited at $4.26 \mathrm{MHz}$ and $4.32 \mathrm{MHz}$ correlates with the weak EHIL shown in Figure 8. According to Figures 8 and 9, while OTSI line gets stronger for $f_{p} \sim 3 f_{c e}$, the NSEE features including DP and IA MSBS lines get weaker. The emissions observed by an SEE receiver include all excited wave vectors, while the EHIL observed by a radar such as MUIR includes only the single wave vector selected by the radar beam direction and transmitter frequency. However, the IA MSBS line being much narrower in bandwidth may be more suitable than the EHIL for temperature estimation. Bernhardt et al. (2009) have shown that by using the matching condition at the UH altitude $\left(\omega_{0}^{2}=\Omega_{c e}^{2}+\omega_{p e}^{2}\right)$ and the quasilongitudinal $(\mathrm{QL})$ approximations to the electromagnetic wave dispersion equations, the ion acoustic sound speed $\left(C_{I A}\right)$ and the electron temperature $\left(T_{e}\right)$ can be estimated from the following expressions:

$$
C_{I A}=\frac{c \omega_{I A}}{2 \sqrt{\Omega_{c e} \omega_{0}}} \sqrt{\frac{\Omega_{c i}^{2}-\omega_{0}^{2}}{\Omega_{c i}^{2} \cos ^{2} \theta-\omega_{0}^{2}}} \sqrt{\frac{\omega_{0}+\Omega_{c e} \cos \theta}{\omega_{0} \cos \theta+\Omega_{c e}}}(1)
$$

$$
T_{e}=\frac{m_{i} c^{2} \omega_{I A}^{2}}{\left(\gamma_{e}+\gamma_{i} / 3\right) 4 \Omega_{c e} \omega_{0}} \frac{\Omega_{c i}^{2}-\omega_{0}^{2}}{\Omega_{c i}^{2} \cos ^{2} \theta-\omega_{0}^{2}} \frac{\omega_{0}+\Omega_{c e} \cos \theta}{\omega_{0} \cos \theta+\Omega_{c e}}(2)
$$

where $\omega_{I A}$ is the measured upshifted ion acoustic frequency in the NSEE spectrum, $\Omega_{c i}$ and $\Omega_{c e}$ are the ion and electron gyro-frequency, $\theta$ is the angle between the wave normal direction and the background magnetic field vector. $\gamma_{e}$ and $\gamma_{i}$ are 1 and 3 , respectively. The previous study by Bernhardt et al. (2009) showed that the estimated electron temperature using IA SBS frequency offset reduced about $500 \mathrm{~K}$ between the two consecutive heating cycle. This was also expected due to the change of the coupling to the plasma by variations in the interaction altitude as well as increased D-region absorption. Another study by Mahmoudian et al. (2014a) has shown the correlation of electron temperature estimated using IA frequency offset excited through the MSBS process at UH resonance layer and the strength of artificial optical emissions. Detailed calculation of $T_{e}$, using IA MSBS lines excited at the reflection altitude will be the subject of future investigation. The narrowband SEE (NSEE) measurement could be a good candidate for locations that ISR facilities are not available, or as a complementary measurement for waves and irregularities that cannot be observed by ISR.

Persistent enhancement of ion lines in the MUIR radar spectrum is observed for pump frequency $4.32 \mathrm{MHz}$. This is consistent with the previous theoretical predictions by Dubois et al. (1993a, b), that that persistent enhancement of UHF diagnostic radar backscatter should be in- 

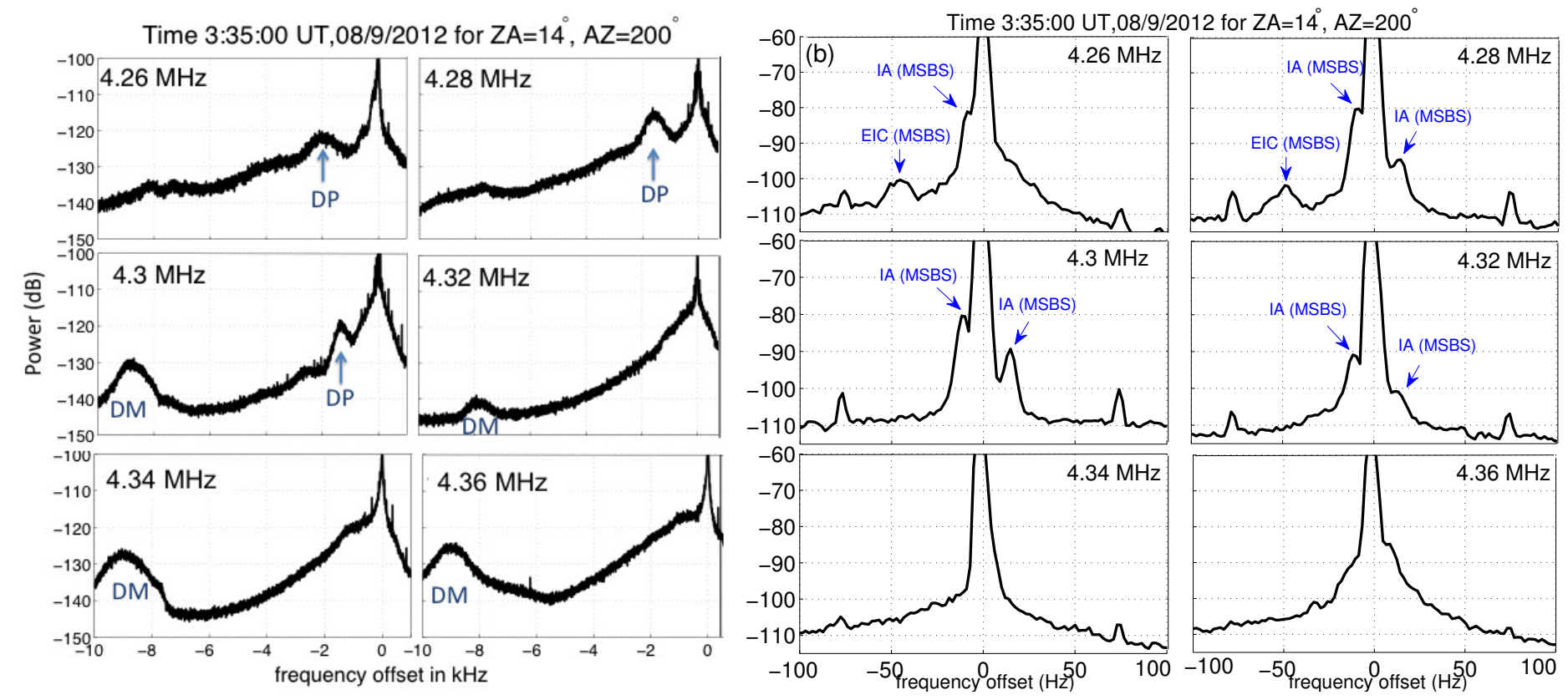

Figure 9: (a) Wideband and (b) narrowband SEE spectra, both corresponding to magnetic zenith (MZ) HF beam and pump frequency variation from 4.26 to $4.34 \mathrm{MHz} .3 f_{c e} \sim 4.31 \mathrm{MHz}$

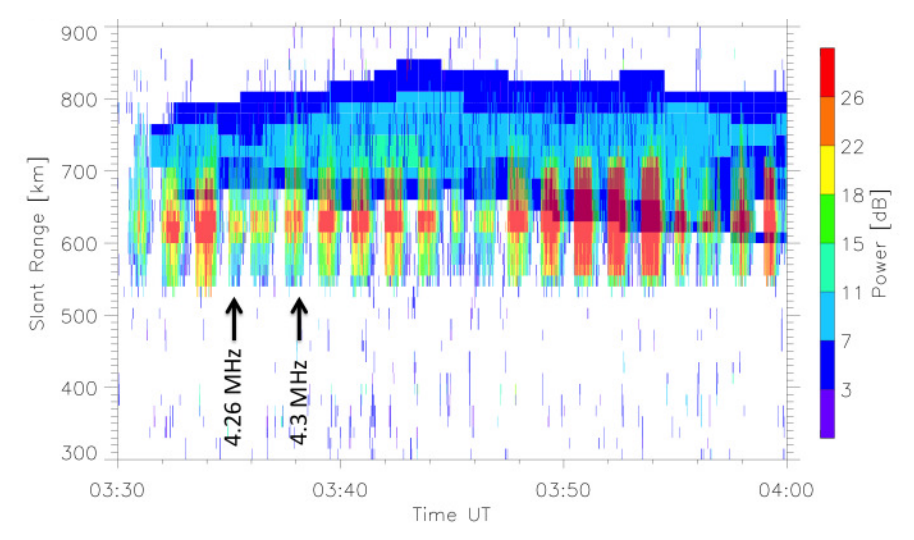

Figure 10: Backscatter observed by the Kodiak radar during ionospheric heating on 9 August 2012. The pump frequency was swept from 4.26 to $4.38 \mathrm{MHz}$ in $0.02 \mathrm{MHz}$ steps. The SuperDARN observations show the suppression of excited FAI for pump frequencies close $3 f_{c e} \approx 4.31 \mathrm{MHz}$, which is consistent with previous observations. duced when the HF pump frequency is slightly lower than $f_{\mathrm{O}} F_{2}$ by a few hundred $\mathrm{kHz}$. According to the ionogram data (not shown here) $f_{\mathrm{O}} F_{2}$ was $\sim 4.33 \mathrm{MHz}$ during this experiment. The decay line is generated at the so-called matching height (Dubois et al., 1993a, b). More refined experimental observations are required to definitively resolve which SEE line most closely correlates with the behavior of the persistent EHIL as observed at $4.32 \mathrm{MHz}$. According to the theory, stimulated Brillouin scattering can cause asymmetry in UHF radar spectrum in such a manner that the upshifted EHIL is enhanced and the downshifted EHIL is suppressed (Fejer et al., 1978). To verify this, a time average of radar spectra shown in Figs 1, 3 and 5 is required.

In order to assess the potential of NSEE measurements as a new diagnostic technique, a comparison between the electron temperatures estimated using narrowband SEE (NSEE) spectral lines, and $T_{e}$ measurements using ISR is required. Unfortunately, the MUIR radar is too small to get any useful ISR measurements outside the HF interaction height. Therefore, to further investigate the potential of NSEE (IA MSBS line) in $\mathrm{T}_{e}$ measurement, future joint NSEE and ISR experiments could be conducted at EISCAT where more suitable ISR facilities are available. Investigation of the correlation of NSEE features and EHILs in the MUIR radar spectra was one of the main goals of the 2011 and 2012 experiments. The frequency offset of IA MSBS lines excited for pump frequency $4.3 \mathrm{MHz}$, shown in Figure 2, is about $10 \mathrm{~Hz}$. The variation of the IA offset and the associated electron temperature with pump frequency, as described in section 3.2, is in agreement with that of the strength of EHIL in MUIR radar spectra. Moreover, the time evolution of the electron temperature (described 
in section 3.1) as the HF pump power increases with time manifests itself as a variation of the IA offset relative to the pump frequency which is an indication of electron temperature enhancement. It should be noted that IA MSBS lines are much narrower than wideband SEE (WSEE) lines and as a result electron temperatures estimated using NSEE line offsets are much more accurate. The detailed comparisons of electron temperatures derived from NSEE and ISR spectra are a topic of current investigation.

The SEE technique has limitations since it does not allow direct observation of the electrostatic (ES) waves necessary for their generation. Therefore, including the data from ground-based radars capable of detecting ES fluctuations can enhance the understanding of plasma processes leading to the generation of SEE. Resonance instability near the UH resonance altitude can produce strongly elongated irregularities and results in enhanced field-aligned scattering of radio waves as stated earlier (Gurevich, 2007). Trapping of UH waves is possible when the amplitude of striations becomes sufficiently large and that may lead to additional mode conversion, heating and generation of thermal cavitons (Grach et al., 1978; Hysell et al., 2010; Samimi et al., 2014) which are the characteristics of the resonance instability that is the nonlinear development of the thermal OTSI. The resonance instability can cause electron temperature enhancement through the absorption of the plasma EM pump wave and lead to an increase of inhomogeneties. The electron temperature increase can enhance the linear process and generate stronger field aligned density striations (FAI). The enhancements of the SuperDARN backscatter spectra near zero Doppler frequency offset was interpreted as FAI due to the thermal OTSI/reson instability excited near the UH resonance layer. FAI generated by UH waves through resonance/thermal OTSI instability has a maximum growth rate of $22 \mathrm{~s}^{-1}$ for irregularity scale sizes of $6 \mathrm{~m}$ (Leyser, 2001). The minimum value of electric field for generating 20-m-scale FAI is around 0.1 $\mathrm{V} / \mathrm{m}$ (Gurevich et al., 1995). The electric field amplitude is estimated to be of the order of a few tenths of $\mathrm{V} / \mathrm{m}$. The Kodiak radar was operating at $9 \mathrm{MHz}$ during this experiment (Figure 7) which corresponds to an irregularity scale-size $17 \mathrm{~m}$, which suggests that the threshold for the excitation of FAI was reached.

The thermal OTSI theory and the linear dispersion relation of UH waves imply that the excitation of small-scale FAI should be suppressed for $f_{0}$ near $n f_{\text {ce, }}$, as is the case for the DM emission. The experimental observations shown in Figures 8 and 9 are consistent with this theory. As can be seen, the DM for pump frequency near $3 f_{c e}\left(f_{p}=4.32\right.$ $\mathrm{MHz}$ ) quenches, which is coincident with the minimum in the intensity of resonant HF scattering off FAI in the Kodiak data. As the DM line became more pronounced at pump frequencies higher than $3 f_{c e}$, stronger Kodiak echoes were observed. The work by Mjolhus (1993) has shown that suppression of FAI for pump frequencies below $n f_{c e}$ is attributed to the excitation of Bernstein modes in addition to UH waves as well as collisionless damping. Ac- cording to Figures 2, 4, 6 and 7, the comparision between the appearence of the NSEE line (IA MSBS) and WSEE with frequency offset $>2 \mathrm{kHz}$, as well as the formation of FAI, shows that there is a correlation between the growth time of FAI and WSEE. This is consistent with the experimental observation that WSEE is directly dependent on FAI growth. SBS NSEE is not dependent on FAI growth as it is a direct mode conversion from the scattered wave.

\section{Conclusions}

A main purpose of this paper is to assess the potential of NSEE as a diagnostic during heating by comparing it with other more established diagnostics such as WSEE, irregularity observations with SuperDARN HF backscatter radar, and MUIR (Modular UHF Ionospheric Radar). The data recorded during two campaigns at HAARP, in 2011 and 2012 is presented. Several diagnostic instruments were used to detect HAARP heater-generated ionospheric irregularities and plasma waves. According to theory, the OTSI can be generated for electric field intensities of about 250 $\mathrm{mV} / \mathrm{m}$ and in a few tens of milliseconds, which is in agreement with the experimental observations. An increase in the excitation time of OTSI was observed as the HF beam was tilted to angles further away from magnetic zenith which is consistent with the theory of the formation of stationary electron density perturbation by the OTSI. During heating, Narrowband SEE (NSEE) lines showed variations, that correlated with enhanced ion lines (EHILs) seen by the MUIR radar. IA MSBS NSEE lines are much narrower than WSEE lines and as a result electron temanqeratures estimated using NSEE line offsets has the potential for improved accuracy. The strength of IA MSBS lines correlates with EHILs in the MUIR spectra spectrum when varying the HF pump frequency near $3 f_{c e}$. Therefore, NSEE could be used for similar diagnostic information, particularly temperature assessment during heating. Additional detailed physics-based modeling of NSEE behavior near gyro-harmonics is expected to provide further diagnostic information/capabilities. It has been noted that future experiments at locations with ISR facilities such as EISCAT, could verify the potential of $T_{e}$ assessment using NSEE and as a complementary measurement to ISR. This work has shown the plausibility of Narrowband SEE (NSEE) as a powerful untapped ionospheric diagnostic which could provide complementary measurements for locations where ISR facilities are not available or as a complementary measurement of waves and irregularities that cannot be observed by ISR. Near term possibilities for developing NSEE as a diagnostic may be further realized at heating facilities such as EISCAT or Arecibo where more suitable ISR facilities currently exist. Recent observations of NSEE at EISCAT (Fu et al., 2015) underscore these opportunities.

Acknowledgements

This work was supported by the National Science Foundation. The work at NRL is supported by the 6.1 research 
program. B.I. and O.V.-C. were supported by ARO grants W911NF-11-0217 and W911NF-10-1-0002. Support for BW was provided by DARPA-DSO under Contract No. HR0011-09-C-0099 and by ONR under Grant No. N0001403-1-0165.

\section{References}

Bernhardt, P. A., Selcher, C. A., Lehmberg, R. H., Rodriguez, S., Thomason, J., McCarrick, M., and G. Frazer (2009), Determination of the electron temperature in the modified ionosphere over HAARP using the HF pumped Stimulated Brillouin Scatter (SBS) emission lines, Ann. Geophys., 27, 4409-4427, doi:10.5194/angeo27-4409-2009.

Bernhardt, P. A., Selcher, C. A. Lehmberg, R. H. Rodriguez, S. P. Thomason, J. F. Groves, K. M. McCarrick, G. J. Frazer(2010), Stimulated Brillouin Scatter in a Magnetized Ionospheric Plasma , Phys. Rev. Lett., 104, doi:16500410.1103/PhysRevLett.104.165004.

Bernhardt, P. A., Selcher, C. A. and S. Kowtha (2011), Electron and ion Bernstein waves excited in the ionosphere by high power EM waves at the second harmonic of the electron cyclotron frequency, Geophys. Res. Lett., 38, L19107, doi:10.1029/2011GL049390.

Blagoveshchenskaya, N. F., T. D. Borisova, M. Kosch, T. Sergienko, U. Brndstrm, T. K. Yeoman, and I. Hggstrm (2014), Optical and ionospheric phenomena at EISCAT under continuous X-mode HF pumping, J. Geophys. Res. Space Physics, 119, doi:10.1002/2014JA020658.

Burton, L. M., J. A. Cohen, R. Pradipta, A. Labno, M.C. Lee, Batishchev O., D.L. Rokusek, S.P. Kuo , Watkins B. J. and S. Oyama (2008), Excitation and diagnosis of cascading Langmuir waves in ionospheric plasmas at Gakona, Alaska Phys. Scr. T132014030.

Chen, F. F., Introduction to Plasma Physics, Springer US, 2012

Cohen, J. A. , R Pradipta, L. M. Burton, A Labno, M. C. Lee, B. J. Watkins, C Fallen, S. P. Kuo, W.J Burke, D Mabius and B.Z See (2010), Generation of ionospheric ducts by the HAARP HF heater, Phys. Scr. 2010014040 doi:10.1088/00318949/2010/T142/014040.

DuBois, D. F., A. Hanssen, A. R. Harvey, and D. Russell (1993a), Space and time distribution of HF excited Langmuir turbulence in the ionosphere: Comparison of theory and experiment, J. Geophys. Res., 98(A10), 17,543 17,567.

DuBois, D. F., A. Hansen, H. A. Rose, and D. Russell (1993b), Excitation of strong Langmuir turbulence in the ionosphere: Comparison of theory and observations, Phys. Fluids B, 5(7), 2616 2622.

Dysthe, K . B., E. Mjolhus, H. L. Pecseli, and K. Rypdal (1983), A thermal oscillating two stream instability, Phys.Fluids, 26, 146.

Fejer, J. A., Rinnert K., and R. Woodman (1978), Detection of stimulated Brillouin scattering by the Jicamarca radar, J. Geophys. Res., 83, 21332136.

Fejer, J. A., Physical processes of ionospheric heating experiments, Adv. Space Res., 8, (1), 261, 1988.

Fu, H., W. A. Scales, P. A. Bernhardt, A. Samimi, A. Mahmoudian, S. J. Briczinski, and M. J. McCarrick (2013), Stimulated Brillouin scatter and stimulated ion Bernstein scatter during electron gyroharmonic heating experiments, Radio Sci., 48, 607616, doi:10.1002/2013RS005262.

Fu, H. Y., Scales, W. A., Bernhardt, P. A., Briczinski, S. J., Kosch, M. J., Senior, A., Rietveld, M. T., Yeoman, T. K., and J. M. Ruohoniemi (2015), Stimulated Brillouin scattering during electron gyro-harmonic heating at EISCAT, Ann. Geophys., 33, 983-990, doi:10.5194/angeo-33-983-2015.

Grach, S. M., A. N. Karashtin, N. A. Mityzkov, V. O. Rapoport, and V. Y. Trakhtengerts (1978), Theory of thermal parametric instability in an inhomogenous plasma, Sov. J. Plasma Phys., Engl. Transl., 4, 737741.

Gurevich, A. V., Zybin, K. P., and Lukyanov, A. V. (1995), Stationary State of Isolated Striations Developed During Ionospheric Modification, Phys. Lett. A 206, 247259.

Huang, J., and S. P. Kuo (1994), Cyclotron harmonic ef- fect on the thermal oscillating two-stream instability in the high latitude ionosphere, J. Geophys. Res., 99(A2), 2173-2181, doi:10.1029/93JA02668.

Huang, J., and S. P. Kuo (1995), A generation mechanism for the downshifted peak in stimulated electromagnetic emission spectrum, J. Geophys. Res., 100(A11), 2143321438, doi:10.1029/95JA02302.

Hughes, J. M., W. A. Bristow, R. T. Parris, and E. Lundell (2003), SuperDARN observations of ionospheric heater induced upper hybrid waves, Geophys. Res. Lett., 30(24), 2276, doi:10.1029/2003GL018772.

Hughes, J. M., W. A. Bristow, and R. T. Parris (2004), SuperDARN observations of spectral enhancements excited during an ionospheric heating experiment, Geophys. Res. Lett., 31, L08808, doi:10.1029/2004GL019613.

Hysell, D. L. and Nossa, E. (2009), Artificial E-region field-aligned plasma irregularities generated at pump frequencies near the second electron gyroharmonic, Ann. Geophys., 27, 27112720.

Hysell, D. L., E. Nossa, and M. McCarrick (2010), Excitation threshold and gyroharmonic suppression of artificial E region fieldaligned plasma density irregularities, Radio Sci., 45, RS6003, doi:10.1029/2010RS004360.

Kuo, S. P. (2003). On parametric instabilities in HF heating of the ionosphere. Journal of Plasma Physics, 69, pp 529-540 doi:10.1017/S0022377803002381.

Leyser, T. B., Thid, B., Derblom, H., Hedberg, ., Lundborg, B., Stubbe, P., and H. Kopka (1989), Stimulated Electromagnetic Emission Near Electron Cyclotron Harmonics in the Ionosphere, Phys. Rev. Lett. 63, 11451147.

Leyser, T. B., B. Thide, H. Derblom, A. Hedberg, B. Lundborg, P. Stubbe,and H. Kopka (1990), Dependence of stimulated electromagnetic emis-sion on the ionosphere and pump wave, J. Geophys. Res., 95(A10),1723317244, doi: 10.1029/JA095iA10p17233.

Leyser, T. B. (2001), Stimulated electromagnetic emissions by highfrequency electromagnetic pumping of the ionospheric plasma, SpaceSci. Rev., 98, 223328.

Leyser, T. B., B. Thidd, M. Waldenvik, E. Veszelei, V. 1. Frolov, S. M. Grach, and G. P. Komrakov, Downshifted maximum features in stimulated electromagnetic emission spectra, J. Geophys. Res., 99, 19555-19568, 1994.

Lundborg, B., and B. Thid (1985), Standing wave pattern of HF radio waves in the ionospheric reflection region 1. General formulas, Radio Sci., 20(4), 947957, doi:10.1029/RS020i004p00947.

Mahmoudian, A., W. A. Scales, P. A. Bernhardt, H. Fu, S. J. Briczinski, and M. J. McCarrick (2013a), Investigation of ionospheric stimulated Brillouin scatter generated at pump frequencies near electron gyroharmonics, Radio Sci., 48, 685-697, doi:10.1002/2013RS005189.

Mahmoudian, A., W. A. Scales, P. A. Bernhardt, A. Samimi, E. Kendall, J. M. Ruohoniemi, B. Isham, O. Vega-Cancel, and M. Bordikar (2013b), Ion gyroharmonic structuring in the stimulated radiation spectrum and optical emissions during electron gyroharmonic heating, J. Geophys. Res. Space Physics, 118, 1270-1287 doi:10.1002/jgra.50167.

Mahmoudian, A., W. A. Scales, P. A. Bernhardt, B. Isham, E. Kendall, S. J. Briczinski, N. E. B. Fuentes, and O. VegaCancel (2014a), Electron gyroharmonic effects on ionospheric stimulated Brillouin scatter, Geophys. Res. Lett., 41, 5710-5716, doi:10.1002/2014GL061050.

Mahmoudian, A.; Scales, W.; Bordikar, M., Bernhardt, P. A. (2014b), Narrowband stimulated electromagnetic emissions (SEE) spectra: A new ionospheric diagnostic technique, General Assembly and Scientific Symposium (URSI GASS), 2014 URSI, vol., no., pp.1,4, 16-23 Aug. 2014.

Mjlhus, E. (1993), On the small scale striation effect in ionospheric modification experiments near harmonics of the electron gyro frequency, J. Atmos. Terr. Phys., 55(6), 907918.

Norin, L., T. B. Leyser, E. Nordblad, B. Thide, and M. McCarrick (2009), Unprecedentedly strong and narrow electromagnetic emissions stimulated by high frequency radio waves in the ionosphere, Phys. Rev. Lett., 065003, doi:10.1103/PhysRevLett.102.065003. 
Oyama, S., B. J. Watkins, S. Nozawa, S. Maeda, and M. Conde (2005), Vertical ion motion observed with incoherent scatter radars in the polar lower ionosphere, J. Geophys. Res., 110, A04302, doi:10.1029/2004JA010705.

Oyama, S.-I., B. Watkins, F. T. Djuth, M. J. Kosch, P. Bernhardt, and C. J. Heinselman (2006), Persistent enhancement of the HF pump-induced plasma line measured with a UHF diagnostic radar at HAARP, J. Geophys. Res., 11, A06309, doi:10.1029/2005JA011363.

Samimi, A., W. A. Scales, P. A. Bernhardt, S. J. Briczinski, C. A. Selcher, and M. J. McCarrick (2012a), On ion gyro-harmonic structuring in the stimulated electromagnetic emission spectrum during second electron gyro-harmonic heating, Ann. Geophys., doi:10.5194/angeo-30-1587-2012.

Samimi, A., W. A. Scales, P. A. Bernhardt, S. J. Briczinski, and M. J. McCarrick (2014), Ion gyroharmonic structures in stimulated radiation during second electron gyroharmonic heating: 2. Simulations, J. Geophys. Res. Space Physics, 119, doi:10.1002/2013JA019341.

Scales, W. A., M. R. Bordikar, A. Samimi, P. A. Bernhardt, S. Briczinski, C. A. Selcher, and M. McCarrick (2011), Observations and theory of ion gyroharmonic structures in the stimulated radiation spectrum during second electron gyro-harmonic heating, General Assembly and Scientific Symposium, URSI, doi:10.1109/URSIGASS.2011.6051126.

Scales, W. A. (2016), Recent advances in stimulated radiation studies during radiowave heating the near earth space environment, Radiation Effects and Defects in Solids Vol. 171, Iss. 1-2, doi: 10.1080/10420150.2016.1155586.

Stubbe, P. (1996), Review of ionospheric modification experiments at Troms, J. Atmos. Terr. Phys., 59, 349368.

Thide, B., H. Kopka, and P. Stubbe (1982), Observation of stimulated scat-tering of a strong high frequency radio wave in the ionosphere, Phys. Rev.Lett., 49, 15611564, doi:10.1103/PhysRevLett.49.1561.

Vaskov, V. V., and A. V. Gurevich (1977), Resonance instability of smallscale plasma perturbations, Sov. Phys. JETP, Engl. Trans., 46,487494 . 